\title{
Multi-scale Interaction of Particulate Flow and the Artery Wall
}

I. Halliday ${ }^{1 *},{ }^{1}$ M. Atherton ${ }^{2}$, C. M. Care ${ }^{1}$, M. W. Collins ${ }^{2}$, D. Evans ${ }^{3}$, P. C. Evans ${ }^{4}$, D. R. Hose

${ }^{3}$, A. W. Khir ${ }^{2,5}$, C. S. König ${ }^{5}$, R. Krams ${ }^{6}$, P. V. Lawford ${ }^{3}$, S. V. Lishchuk ${ }^{1}$, G. Pontrelli ${ }^{7}$, V. Ridger $^{8}$, T.J. Spencer ${ }^{1}$, Y. Ventikos ${ }^{9}$, D. C.Walker ${ }^{10}$, P. N. Watton. ${ }^{9}$

* Corresponding author: Tel.: ++44 (0)114 2253045; Fax: ++44 (0)114 2253045; Email:

i.halliday@shu.ac.uk

1 Materials and Engineering Institute, Sheffield Hallam University, Sheffield UK

2 School of Engineering and Design, Brunel University, London UK

3 Medical Physics Group, Department of Cardiovascular Science, University of Sheffield, Sheffield UK

4 BHF Cardiovascular Sciences Unit, National Heart and Lung Institute, Imperial College, London UK

5 Brunel Institute for Bioengineering, Brunel University, London UK

6 Department of Bioengineering, Imperial College London, London UK

7 CNR Rome, Tor Vergata, Rome, Italy

8 Cardiovascular Research Unit, University of Sheffield, Sheffield UK

9 Institute of Biomedical Engineering, Department of Engineering Science, University of Oxford,

Oxford UK

10 Department of Computer Science, University of Sheffield, Sheffield UK

\begin{abstract}
We discuss, from the perspective of basic science, the physical and biological processes which underlie atherosclerotic (plaque) initiation at the vascular endothelium, identifying the widely separated spatial and temporal scales which participate. We draw on current, related models of vessel wall evolution, paying particular attention to the role of particulate flow (blood is not a
\end{abstract}


continuum fluid), and proceed to propose, then validate all the key components in a multiplycoupled, multi-scale modeling strategy (in qualitative terms only, note). Eventually, this strategy should lead to a quantitative, patient-specific understanding of the coupling between particulate flow and the endothelial state.

Keywords: Multi-scale, Microcirculation, Endothelium, Wall shear stress, Glycocalyx

\section{Introduction}

Atherosclerotic plaque rupture and vessel obstruction together produce the highest mortality in the western world. Although it is known to be lipid-driven, inflammation and blood flow have generated recent interest as alternative or complementary explanations for the formation of atherosclerotic lesions (plaques) $(1,2)$. The role of flow, long evident in the observation that plaques occur near arterial side branches, bifurcations, or bends (where flow rates and shear are low, disturbed or oscillatory) is coupled to other processes by activation of the vascular endothelium, which when quiescent, inhibits leukocyte adhesion, platelet aggregation and exhibits anti-inflammatory, anti-proliferative and anti-oxidant characteristics. A dysfunctional endothelium is a key mediator in the pathogenesis of arterial disease $(3,4)$. In this capacity, the geometrical conformation of its constituent cells (see section 2), which, in part defines our endothelial state, is a very significant factor.

As this article proceeds, we will seek to show that the physics and biology of these mechanisms lies on disparate spatial and temporal scales, which calls for a particular approach to the problem of developing a quantitative model. Here, we describe, and contextualize our recent progress towards this end, namely a complex, multi-scale, in silico model of plaque initiation founded in basic science, paying particular attention to flow. Section 2 (and the references therein) provides 
background to the biological and physical mechanisms, whilst emphasizing their spatial-temporal scales. Sections 3 and 4 review issues around the coupling of scales in physiological models and explain our multi-scale model, which seeks self-consistently to couple the endothelium state with previously unresolved flow modalities. In section 5 we present, discuss and contextualize our results and in section 6 present our conclusions and intentions for future work.

\section{Background}

The healthy arterial wall (Figure 1) contains endothelial cells (ECs), smooth muscle cells and fibroblasts. The vascular endothelium, which regulates vascular inflammation, consists of an EC monolayer on a collagen basement membrane. It serves to regulate the capture and subsequent infiltration of, among others, inflammatory cells (eg. leukocytes) into the underlying tissue. Multiscale, flow processes couple this function directly, via flow stresses, and indirectly, by chemical convection-diffusion, to plaque formation. Flow is also known to affect endothelial expression of several adhesion proteins (which govern capture and rolling of leukocytes on the endothelial surface -see below), it determines the distribution of leukocytes and erythrocytes within the lumen (margination) (5) and, crucially, wall shear stress (WSS) is known to influence ECs' morphology (with implications for leukocyte ingress, at ECs' exposed boundaries) as well as gene expression. In turn, wall topography must "two-way couple" to WSS. Phenomenologically, this coupling (of EC morphology with WSS) manifests itself in increasing WSS with changes in EC conformation (6): in vitro, ECs elongate, changing from a polygonal, 'cobblestone’ morphology to flattened, elongated ovoids (Figures 2a, 2b), the degree of elongation correlating with the magnitude of the shear stress (7-10). Note that WSS type, too, is relevant- under oscillatory shear, cultured cells retain a polygonal shape (8) and in vivo, ECs have been shown to orientate to the predominant local flow direction (11), perpendicular to the direction of cyclic stretch $(12,13)$. Such observed behavior 
should, of course, emerge from a correct and self-consistent model coupling the endothelial state with flow.

Before proceeding with a wider discussion of WSS effects, we briefly return to leukocyte attachment, modelled on rolling. During leukocyte attachment, similarly structured P- or L-type selectins (on leukocyte microvilli and ECs) mediate capture, in a process which may be modeled on the surface rolling of an incompressible drop with regulated wetting $(5,14)$. Longer periods of endothelial stimulation increase adhesion factor density, increase protein-protein interaction and so slow rolling velocity, under the influence of E-selectin (15), exposing a leukocyte to endothelial chemoattractants, resulting in firm adhesion.

In normal human arteries, WSS ranges between 10 and 70 dynes $/ \mathrm{cm}^{2}(1-7 \mathrm{~Pa})$. Ideally, ECs elongate and align with flow, integrin distribution is reorganized, improving EC adhesion, and antithrombic and vasoactive agents (nitric oxide (NO), endothelium-derived hyperpolarizing factor, prostacyclin and endothelin) are produced $(16,17)$ to act on the vascular smooth muscle cells, which regulate the diameter of the vessel and so control local blood flow rate (18). Local regulation of vessel diameter helps to maintain WSS within the normal range. As we have noted, higher and lower values of WSS and of oscillating shear index (OSI) (the latter measures oscillations in flow, and is related to the particle residence times) are found at specific sites in the circulation, associated with changes in geometry (e.g. an anastomosis, stenosis or bifurcation), the lowest values occurring where flow becomes unstable in separation or recirculation zones. Specific levels of WSS are believed to result in increased levels of the biomarkers associated with inflammatory pathways. NO is produced throughout the body but, in the vascular endothelium, its action is especially important, for there it produces flow dependent, vasodilatation, an anti-inflammatory effect inhibiting leukocyte adhesion and NO produced in areas of high WSS (>1.5Pa) inhibits thrombus formation in areas of low WSS. So it is that abnormal production of NO, in disease, adversely affects blood flow and other vascular functions. For example, the isolated flow inside an aneurysm 
is often dominated by low WSS. When the latter is below 0.5Pa, this produces a local environment of extreme thrombus formation (19) and low WSS at the aneurysm fundus can trigger a biological response which initiates rupture and internal hemorrhage (20) when high WSS is present.

Historically, high WSS is considered atheroprotective (21) and low WSS is associated with atherogenesis (3). Several studies suggest that oscillatory shear is also detrimental; eg. Ku et al. (22), correlate atherosclerotic plaque location with areas of low and oscillating WSS in the human carotid bifurcation. An additional level of complexity is demonstrated by the results of in vitro studies indicating that ECs sense temporal and spatial gradients of WSS (23-25). Recent evidence suggests that WSS also influences plaque composition. Vulnerable plaque has been associated with low WSS in mice and pigs and high WSS in rabbits and man. To reconcile these data, it has been suggested that low shear stress induces endothelial dysfunction, while additional, high WSS on this dysfunctional endothelium is needed to convert a plaque to a vulnerable plaque $(26,27)$.

In the substantial literature on the influence of WSS on ECs, most data has been obtained from in vitro experiment using rigid flow chambers and well-characterized flows, when WSS can be calculated with a reasonable level of confidence. In vivo, the situation is more complex and the WSS values estimated represent a mean over a considerable area of the vessel wall and, hence, do not describe individual cells. This approximation may be reasonable, for there is evidence of significant interaction and intercellular signaling between ECs (28), but the length-scale of any response homogenization remains unclear. Additional questions arise when one considers the micro-scale and then the mesoscale constitution of blood, as follows.

Steady haemodynamic flow approximates the real experience of ECs, which must view the flow of the blood as motion of a high volume fraction particle suspension, comprised (at its simplest, we stress), of erythrocytes and incompressible plasma. On this view, necessarily unsteady stresses imparted, on the endothelium, have contributions from fluid stresses and lubricated "particle impacts" and true WSS must have a spatial/temporal structure which is entirely neglected by the 
continuum haemodynamic approximation. The unstudied, statistical properties of such "real” WSS distributions will depend, inter alia, upon correlations with the large-scale vessel geometry and the local micro-geometry (ie. the instantaneous conformation of ECs). They will also depend on the microscopic surface structure of the erythrocytes as follows.

The flow boundary conditions which describe the layer of fluid "sandwiched" between an advected erythrocyte and an EC are not necessarily no-slip, Dirichlet conditions. Instead, Navier-type conditions, which model the microscopic properties of the membrane-bound macromolecular coating (29) found on the ECs' luminal surface, the so-called glycocalyx, must be considered. Comprising sulphated proteoglycans, hyaluronan and glycoproteins, the glycocalyx has a net negative charge and it binds blood-born molecules such as plasma proteins, enzymes cytokines, water and other molecules with cationic sites in a layer $~ 500 \mathrm{~nm}$ thick (30). Interestingly, the thickness of this layer is reduced at sites where atherosclerosis is initiated and its influence on WSS at the sub-haemodynamic scale may be very important.

In summary, the physics, biology and the scales of the coupled processes outlined in this section urge a multi-scale approach to a quantitative understanding of endothelial behavior, based on interfacing a range of appropriately adapted, two-way coupled computational strategies. We will discuss our approach in section 4: first we review that current work which provides its basis.

\section{Coupling vessel evolution to flow}

$\mathrm{Xu}$ and Collins (31) showed that, to be meaningful clinically, traditional computational fluid dynamics (CFD) based upon discretization of the Navier-Stokes and continuity equations and an appropriate constitutive equation for blood (approximated as a continuum), should have the potential to address: a] the time-dependent pulsatility of blood flow, b] the non-Newtonian character of blood (ie the non-linear relationship between viscosity and shear stress), c] the 3D 
geometry of the arterial system, and d] its time-dependence, due to the distensibility of the arterial wall. Several data sources are therefore essential to the development of a closed computational model; a description of the flow boundary conditions, a description of blood viscosity and appropriate sets of validation data, against which key features of the model's output might be tested. In vitro experiments by Moravec and Liepsch (32) were used in this capacity (33), later in vivo canine bifurcation data (34) were added, before the model was extended to accept clinical Magnetic Resonance (MR) data for inlet boundary conditions (35). Finally, a number of alternative fluidstructure-interaction (FSI) models were successfully validated using Womersley’s data (36). CFD codes have now been combined with solid mechanics codes to give fluid-structure interaction (FSI) capability but, for cardiovascular vessels, there remains a paucity of data on the constraints on vessel wall motion in vivo. One solution is to use time series image data to describe wall motion. In a very recent study characterising the haemodynamics of the human superior mesenteric artery (SMA), a fully transient, CFD model was constructed of a segment of the abdominal aorta and SMA. The geometry was based on dynamic MR imaging data and boundary conditions (flow), were determined from phase contrast MR velocity measurements (37). The abdominal aorta was shown to be a principal site for the development of atherosclerotic lesions (38) as are the immediate proximal (coeliac) and distal (renal) branches. (see the @neurIST project (http://www.aneurist.org)). The resulting OSI map for the model is shown in Figure 3a. OSI is, in general, lower in the SMA than in the aorta. The only area of high OSI in the SMA is at the root of the vessel as it leaves the aorta. This correlates with clinical observations for atheroma. Figure 3b illustrates the time varying WSS for two areas of the vessel wall exposed to a high or low level of OSI.

Tissue remodeling and endothelium signaling have been incorporated into coupled simulations. The evolution (inception, growth and rupture) of cerebral aneurysms provides an illustrative example of a class of models which have applications to disease evolution, as well as to the evaluation of 
competing theories. The first modeling stage is to estimate the haemodynamic loads (pressure and WSS) acting on the aneurysm and parent artery, using a finite volume CFD technique. The distribution of WSS on the inner surface of the aneurysm is computed and used to model a hypothetical endothelial response which subsequently signals the smooth muscle cells and fibroblasts and leads to a remodeling and growth or atrophy of the elastin and collagen in the arterial wall. This results in changes in the mechanical properties of the aneurysm and its geometry adapts (to maintain mechanical equilibrium), a finite element method being used to solve the updated deformation field. Of course, a new geometry changes the haemodynamics and, hence, the WSS distribution, so an updated flow field is solved and the process iterated, the computational cycle being repeated until the aneurysm stabilizes in size, or until the loads imposed exceed the load-bearing capacity of the wall, leading to rupture.

Such integrative models can be used to relate growth and remodeling of the arterial wall to the haemodynamic stimuli acting on ECs. For instance, the magnitude of the spatial WSS gradient (WSSG) has been proposed as one possible haemodynamic parameter which is correlated with regions of pathological remodeling (39). As an aneurysm adapts the WSSG spatial distribution (see Figure 4) acting on the endothelium will change substantially, the subsequent effects on tissue remodeling being of critical importance.

In studies as the two discussed above, the models' uncertainties are, ideally, removed by resolving the cellular-scale (with proper account of the issues raised in section 2). In the current context, such a step would require one to depict interactions between ECs and flow and demands explicit representation of blood cells and plasma. CFD-based haemodynamics treats blood flow as a flow of continuous fluid and the vascular endothelium as the relevant, geometrically complex, velocity boundary condition. In reality, a local endothelial state emerges from encounters between individual (but interacting) ECs in a Newtonian plasma which advects discrete particles, whose encounter with individual ECs is determined by local geometry, flow rate and cell concentration. 


\section{Multi-Scale Models of Vessel Evolution}

Work such as that outlined in section 3 couples independent, essentially macro-scale models to form a closed description. As we have remarked, however, some situations require closure from the cellular scale: put another way, some situations require the simultaneous use of microscopic and mesoscopic models.

Investigations of the interaction of intravascular devices with the arterial wall, specifically; the Intra Aortic Balloon Pump (IABP) and vascular stent demonstrate a need to address smaller scales in general and the endothelium (and possibly the glycocalyx) in particular. The IAB is used to support a failing heart and is usually inserted via the iliac artery and placed in the abdominal aorta. The balloon is actively inflated (during early diastole) and deflated (during late diastole) every, everyother or every third cardiac cycle, aiming to increase coronary flow and reduce left ventricular afterload. Inevitably, the balloon contacts the inner wall of the aorta, which could lead to balloon rupture, especially in atherosclerotic patients with plaques lining the inner wall of the aorta. As the balloon approaches the endothelium during inflation, a volume of blood is displaced proximally and distally, generating as yet un-quantified WSS. When the balloon moves away from the endothelium during deflation, it will generate micro pressure differences which may impose stretching (pulling) stresses on the ECs. Very high spatial resolution is required fully to interpret these effects at the cellular level. Such micro-scale information is also important in evaluating the performance of vascular stents, which very effectively dilate a stenosed artery to restore flow but at the near-wall micro-scale, their constituent struts (side/diameter 100 $\mu \mathrm{m}$ ) apply stresses to the endothelium and glycocalyx, disturbing local flow and causing further interaction with endothelial topography, Noting the need for improved mechanical models (based eg. upon accurate constitutive laws) to account for tear, macroscopic CFD model, like those of Raedelli et al. capture key details of flow 
but cannot resolve crucial cellular and sub-cellular scales (40). The interaction of (portions of) a stent with individual blood cells, ECs and the glycocalyx is too demanding a problem for conventional CFD.

Arguments of self-consistency, alone, require any model which resolves individual ECs also to resolve the cellular structure of blood. Notwithstanding our comments in section 2, on the unreliability, at the cellular micro-scale, of the concept of steady WSS, the CFD analyses described in this section treat blood flow as flow of a continuous fluid and represent the vascular endothelium as a constant, locally smooth velocity boundary condition. We therefore summarize this section by repeating our core hypothesis. Observations urge that a self-consistent, quantitative understanding of the relationship between atherosclerotic initiation and flow (more specifically WSS) must encompass the discrete, cellular scale of erythrocytes and ECs, explicitly represent the continuum, Newtonian, liquid (plasma) and, finally, refer to a model of the lubricating or cushioning effect of the microscopic glycocalyx, to which structure we shall shortly return.

\section{Multi-scale, multi-physics modeling}

We are not the first to appreciate that the enormous increases in computing storage and power seen over the last decade permit corresponding advances in the modelling of engineering and physiological systems. Recently, the NanoInterface Consortium has deployed coupled models ranging from molecular dynamics, through medium-scale models (addressing roughness and filler particles in polymers) to macro-scale models to predict de-lamination. The range of spatial scales is accompanied by a wide range of time scales (for molecular dynamics-picoseconds, for polymer material behaviour- seconds to hours). The well-known Physiome Project strikingly applies such an engineering multi-scale approach, combined with a multi-physics approach (41), to cardiac modelling (42) in which the whole heart is resolved into 'a hierarchy of simplified, smaller models 
capable of both 'interaction and individual tuning' (42), paving a way whole organ simulations which run in real time (as opposed to current whole-cardiac models, which take computational hours for 'seconds of real heart-time'). Multi-scale, multi-physics cardiac modelling has also been undertaken by Liu et al (43) whose Figure 1 elegantly describes the various levels of model, ranging from the $0.1 \mathrm{~m}$ organ scale, down to the molecular $1 \mathrm{~nm}$ scales characterising 'focal adhesion complex'.

Clearly, human physiological systems subsume molecular and cellular, biological and physical spatial and temporal scales, coupling the latter across orders of magnitude, necessitating a multiscale approach.

For the interaction of explicitly resolved blood flow and the vessel wall, mediated by the vascular endothelium, an appropriate multi-scale modeling paradigm is effectively defined in the scale separation map (SSM) of Figure 5. There, the system is resolved into a closed set of coupled, single-scale models, or sub-systems, each mapped according to its characteristic spatial and temporal scales. Evans et al. (44) have shown how this analysis is developed for in-stent restenosis, in the coronary artery (see also www.complex-automata.org, (45) and Liu et al. diagrammatically depict, more qualitatively, key multiple-scale phenomena occurring in the cardio-vascular system (46). In fact, the SSM exemplar of figure 5 is not, in practice, definitive. The placement of, and degree of, resolution used in each sub-system reflects the extent of the modeled domain and the availability of quantitative data; the need to close the model requires one recursively to apply the multi-scale approach.

In section 3 we have shown that the study of OSI (temporal variation in WSS, recall) has added to consistency of CFD predictions with clinical data for atheroma in the abdominal aorta. In aneurysm studies, WSSG is promulgated as a significant parameter for remodelling of the arterial wall. In other words the 'research vector' lies in the direction of studying the length and time scale changes in WSS. The same message may be drawn from section 4, where unanswered questions about the 
IABP and stenting require the study of smaller scales. This observation is confirmed by two recent in vivo studies of WSS by Reneman et al. $(47,48)$, and the in vivo review of Venneman et al. (49). It is candidly admitted that ultrasound and MRI cannot measure velocities closer to the wall than 250-300 and 1000-1200 micrometres respectively (47), which range fails to resolve the glycocalyx (with, recall, characteristic thickness $\approx 100 \mathrm{~nm}$ ). Ultrasonics, therefore, cannot answer a fundamental question as to whether ECs are not seeing WSS' (47) or are 'protected against high flow shear rates that may rupture the cells' (49). While CFD models can give good macroscopic comparisons with in vivo data (for example, 34) they are completely unable to address these issues. It is not so much that they are incorrect, as that they cannot a) resolve these scales and b) the individual cells of both the blood itself and the EC morphology. To achieve a fuller, self-consistent and properly founded understanding of atherosclerosis, these questions must be addressed. It is, therefore, essential that a multi-scale model be founded, insofar as flow is concerned, on a simulation technique which is able efficiently to handle many advected Lagrangian particles (erythrocytes), to accommodate geometrically complex boundary conditions (see below) and multi-physical effects and to facilitate multi-component flow extensions (to model multiple leukocytes and their attachment). The overall demands of the problem suggest a need for parallelizable algorithms. Lattice Boltzmann equation (50) method (LBE) is a meso-scale technique, which is based on a particle distribution function description, which answers all these needs. In particular, when appropriately optimised, LBE has been shown to be able to simulate Lagrangian particulate flow systems over a range of particle volume fractions, in geometries of complexity comparable with that under consideration and on widely available computational platforms $(51,52)$. Figure 6 shows an instantaneous WSS distribution over the boundary of a rectangular cross-section duct at $R e=10$, obtained from a pressure-driven, suspension flow of inelastic spherical "erythrocytes", at hematocrit concentration (40\%), in what would be, in the continuum hemodynamic approximation, the steady state. Clearly, the instantaneous spatial distribution of the WSS component $\eta \partial_{y} u_{x}$ reflects the distribution of 
Lagrangian particles immediately above the boundary. In the data of figure 6, the micro-scale effect of the glycocalyx is modelled crudely, simply by adding to the Lagrangian rigid spheres (each of radius 6 simulation lattice units), and to the duct surface, a surface exclusion region, which has an effect analogous to that of a lubrication force. It should be stressed that a more sophisticated, mesoscale representation of glycocalyx effects would be inconsistent with the simplicity of the smooth boundary representation used for figure 6 , which clearly lacks any reference to the conformation of the EC layer.

The conformational morphology of the EC layer is, however, not beyond the scope of the coupled LBE models we advance here. Figure 7 shows the instantaneous distribution of WSS component $\eta \partial_{y} u_{x}$ over a porcine endothelial surface (bottom surface in figure), calculated for a system comprised of incompressible, Newtonian fluid and a single, advecting "erythrocyte", modelled in the data of figure 7 as an incompressible, immiscible drop with viscosity contrast and interfacial tension. Accordingly, the flow data are $R e=1.2$, Weber number $W e=0.225$ and capilliary number, $\mathrm{Ca}=0.1875$ ). In figure 7 , flow is induced by a pressure gradient in the $x$-direction but the velocity field is periodic in both the $x$ and $y$ directions (53). The simulation is symmetric in the upper plane $z$ $=$ constant, which corresponds to the equator of the drop, and the geometrically complex endothelial surface boundary, in form of a generalised shape $(54,55)$ derived from an electron microscope image of porcine endothelial coronary artery tissue (56), was implemented in out LBE model using a modified form of continuous bounce-back boundary conditions. A region of reduced WSS over the endothelial surface is predicted (not postulated, note) beneath the particle. Such a spatialtemporal WSS fluctuation is, by definition; absent when blood is modelled as a continuum fluideven were a perfect constitutive relation to be modified to account for eg. margination. It is straightforward and computationally inexpensive (both in term of execution speed and memory) to introduce multiple deformable particles, at hematocrit concentrations, and to couple species convection-diffusion into the LBE simulation which generated the data of figure 7 (53). 
However, to account for the conformational response of the ECs to the WSS distribution and, note, to possible particle impact, requires rather more work. To capture this central aspect of the physiological response we propose the use of agent based models (ABMs- see below), which may be readily two-way coupled to flow, when the latter is computed using LBE, in order to animate the endothelial surface (note, it is anticipated that the temporal scale of this process is long, compared with that of the fluctuations in the flow modes).

ABMs have been successfully applied to problems similar to that discussed here, which are defined in figure $5(57,58)$. (Strictly, figure 5 refers to a complex cellular automata (CA) model, in which each component is a CA: then the ABM is a single component representing smooth muscle cell migration and proliferation.) A software agent is an intuitive computational representation of a real world entity applicable to any system where emergent behavior results from complex interactions between many individuals. Vascular endothelial behavior is an emergent property of the responses and interactions of individual ECs, individually represented by simple logical rule sets which determine the responses of individual software agents to biological and physical stimuli alike. Applying ABMs to endothelial tissue resolves the heterogeneity of individual ECs- each EC is permitted to react differently, depending on its gene/protein expression and differences in the local flow field. Sub-models of signaling processes, coupled to flow, extend the logical rule sets, adding further scales and complexity.

It is appropriate to conclude this section by highlighting one final advantage of our multi-scale methodology. Micro-scale experimental data which certainly subject to error but which is unambiguous in its meaning defines the meso-scale model's emergent rheology. Put another way, dynamic and stationary visco-elastic properties of healthy and diseased RBCs have now been measured using a range of techniques, including laser diffractometry, atomic force microscopy and microfluidic devices: this body of basic science data may be used to control RBC membrane properties, removing (validations notwithstanding) the need for a good deal of empiricism in 
calibrating the resulting models.

\section{Conclusion}

Currently, complex simulation chains which facilitate computational fluid dynamics analyses of models, developed from patient-specific geometries, are capable of providing information which, because of the limitations of current investigative techniques (in terms of resolution or the need for direct visualisation, by microscopic imaging of fluorescent particles, for example), is not available from clinical studies or experiment. The ultimate aim is to produce systems which couple haemodynamics with models of biological phenomena, at multiple scales, to test hypotheses or predict disease/intervention outcomes; the potential power of such models is now recognised.

We have argued that a closed multi-scale model of atherosclerotic initiation should capture interactions between representative numbers of explicitly modelled (in the first instance), endothelial cells, Newtonian plasma and the glycocalyx. The spatial and temporal fluctuations apparent in figures 6 and 7 help to identify the longest wavelength of course-graining implicit in the continuum haemodynamic approximation and indicated the need, certainly in our applications, explicitly to resolve spatial and temporal fluctuations of wall shear stress. Figure 6 also demonstrates that the lattice Boltzmann equation method can provide such data.

We have pointed-out that, to complete such a multi-scale model of atherosclerotic initiation, there is a need to model ingress of such atherosclerotic precursors such as leukocytes, which occurs at exposed endothelial cell boundaries. Implicitly, it is therefore necessary to impose geometrically complex, Dirichlet, boundary conditions, corresponding to the conformation of endothelial cells. The evolution of this boundary, we have argued is governed by wall shear stress and species mass transport (convection-diffusion), in coupled processes centred-on meso-scales. While this paper focuses on the role of fluid, it is well recognised, as we have repeatedly stressed, that arterial regulation itself involves inter alia the endothelial cell, wall mechanics and nitrous oxide behaviour. 
In fact an engineering-style control loop has been proposed ((59), updated as (60)) for the overall local regulation process. This evolution of the endothelial state can feasibly be represented by coupled agent-based models, predicated on the micro-scale. But micro-scale information must also be coupled-into the model.

Progress of our model depends on course-grain molecular dynamic studies of effects emergent at the cellular or mesoscale, of the cellular glycocalyx layer. It is currently impossible to resolve cellular-scale flow modalities and (albeit course-grained) molecular scales with one technique. One means of representing the influence of the glycocalyx, is by modelling data from micro-scale studies, to find an effective slip boundary condition, after eg. reference (14).

Several exciting initiatives are poised to accelerate progress and uptake of multi-scale modelling. The rationale of the international Physiome Project describes the need for multi-scale modelling in almost exactly the same terms as we have used. 'Developing models of physiological processes that encompass both multiple physics.... and multi-scale aspects is very difficult and has been achieved in few cases.' However, the framework is being developed (41). Our aspirations in the work reported here are coincident.

In closing, it is worth remarking that, in recognising the potential of computationally-based tools for the simulation of human physiology and disease-related processes, the European Union is currently funding collaborative projects directed towards the development of patient-specific computer models for personalised and predictive healthcare, under the banner of the Virtual Physiological Human (61).

\section{References}

1. Libby P. Nature 2002; 420 (6917) pp868.

2. Cunningham K.S., Gotlieb AI. Lab Investig. (2005) 85 (1) pp9. 
3. Bonetti, P. O., Lerman, L. O., and Lerman, A. (2003) Arterioscler Thromb Vasc Biol 23(2), pp168.

4. Caro, C. G., Fitz-Gerald, J. M., and Schroter, R. C. (1971) Proc R Soc Lond B Biol Sci 177(46), pp109

5. Ridger, V., Krams, R., Carpi, A. and Evans, P. C., Biomedicine and Pharmacotherapy 62 (2008) pp536.

6. Sato, M., Ohshima, N., and Nerem, R. M. (1996) J Biomech 29(4), pp461.

7. Barbee, K. A., Davies, P. F., and Lal, R. (1994) Circ Res 74(1), pp163.

8. Helmlinger, G., Geiger, R. V., Schreck, S., and Nerem, R. M. (1991) J Biomech Eng 113(2), pp123.

9. Levesque, M. J., Liepsch, D., Moravec, S., and Nerem, R. M. (1986) Arteriosclerosis 6(2), pp220

10. Levesque, M. J., and Nerem, R. M., (1985) J Biomech Eng 107(4), pp341

11. Malek, A. M., Alper, S. L., and Izumo, S., (1999) Journal of the American Medical Association 282(21), pp2035

12. Chien, S., (2007) Am J Physiol Heart Circ Physiol 292(3), H pp1209.

13. Owatverot, T. B., Oswald, S. J., Chen, Y., Wille, J. J. and Yin, F. C., (2005) J Biomech Eng 127(3), pp374

14. Spencer, T. J., Hollis, A. P., Halliday, I. and Care, C. M., Proc. Brunel Conference (2009)

15. Ley K, Allietta M, Bullard DC, Morgan S., Circ Res (1998) 83(3) pp287.

16. Korenaga, R., Ando, J., Tsuboi, H., Yang, W., Sakuma, I., Toyo-oka, T., and Kamiya, A. (1994)

Biochem Biophys Res Commun 198(1), pp213.

17. Malek, A., and Izumo, S., (1992) Am J Physiol 263(2 Pt 1), C pp389.

18. Levick, J. R. (2003) An introduction to cardiovascular physiology, Arnold, London.

19. Liou, T-M., Li, Y-C., 'Effects of stent porosity on hemodynamics in a sidewall aneurysm model.‘ (2008) Journal of Biomechanics 41 (5), 1174-1183 
20. Utter,B., Rossman, J.S., (2007) Numeric simulation of saccular aneurysm hemodynamics: Influence of morphology on rupture risk. Journal of Biomechanics 40 (12), 2716-2722.

21. Carlier, S. G., van Damme, L. C. A., Blommerde, C. P., Wentzel, J. J., van Langehove, G., Verheye, S., Kockx, M. M., Knaapen, M. W. M., Cheng, C., Gijsen, F., Duncker, D. J., Stergiopulos, N., Slager, C. J., Serruys, P. W., and Krams, R. (2003) Circulation 107(21), pp2741. 22. Ku, D. N., Giddens, D. P., Zarins, C. K., and Glagov, S. (1985) Arteriosclerosis 5(3), pp293.

23. LaMack, J. A., Himburg, H. A., Li, X. M., and Friedman, M. H. (2005) Ann Biomed Eng 33(4), pp457

24. Tardy, Y., Resnick, N., Nagel, T., Gimbrone, M. A., Jr., and Dewey, C. F., Jr. (1997) Arterioscler Thromb Vasc Biol 17(11), pp3102.

25. White, C. R., Haidekker, M., Bao, X., and Frangos, J. A. (2001) Circulation 103(20), 2508-2513

26. Cheng, C., Tempel, D., van Damme, L., van Haperen, R., Krams, R., and de Crom, R. (2005) Circulation 112(17), 347.

27. Chatzizisis, Y.S., Coskun, A.U., Jonas, M., Edelman, E.R., Feldman, C.L., and Stone, P.H. (2007) JACC 49(25), 2379-2393

28. Demer, L. L., Wortham, C. M., Dirksen, E. R., and Sanderson, M. J. (1993) Am J Physiol 264(6 Pt 2), H pp2094.

29. Hollis, A., Spencer, T.J., Halliday, I., and Care, C.M., (2010) Med. Eng. And Physics

30. Weinbaum, S., Tarbell, J. M., and Damiano, E. R. (2007) Annu Rev Biomed Eng 9, pp121.

31. Xu, X. Y., and Collins, M. W. (1990) Proc Inst Mech Eng [H] 204(4), pp205

32. Moravec, S., and Liepsch, D. (1983) Biorheology 20(6), pp745

33. Collins, M. W. and Xu, X. Y. (1990) A predictive scheme for flow in arterial bifurcations: comparison with laboratory measurements. In: al., F. M. e. (ed). Biomechanical Transport Processes Plenum Press.

34. Xu, X. Y., Collins, M. W., and Jones, C. J. (1992) J Biomech Eng 114(4), pp504. 
35. Long, Q., Xu, X. Y., Collins, M. W., Griffith, T. M., and Bourne, M. (1998) Crit Rev Biomed Eng 26(4), pp227.

36. Xu, X. Y., Collins, M. W., and Jones, C. J. H. (1997) Adv. Eng. Softw. 28(6), pp365.

37. Jeays, A. D., Lawford, P. V., Gillott, R., Spencer, P., Barber, D. C., Bardhan, K. D., and Hose, D. R. (2007) J Biomech 40(9), pp1916.

38. Roberts, J. C. J., Moses, C., and Wilkins, R. H. (1959) Circulation 20(4), pp511.

39. DePaola, N., Gimbrone, M. A., Jr., Davies, P. F., and Dewey, C. F., Jr. (1992) Arterioscler Thromb 12(11), pp1254.

40. Radaelli, A. G., Sola, T., Vivas, E., Mellado, X., Guimaraens, L., Cebral, J. R., and Frangi, A. F. (2007) Combined clinical and computational information in complex cerebral aneurysms: application to mirror cerebral aneurysms. In: Manduca, A., and Hu, X. P. (eds). Proc. SPIE Medical Imaging 2007: Physiology, function, and structure from medical images.

41. Cheng,L.K., Hunter,P.J., Mithraratne,K., Pullan, A.J., Remme, E.W., Reynolds, H.M., Smith, N.P., and Yassis, R. Clinical applications of Physiome Project models, pp 25,

Repair and Redesign of Physiological Systems, (2008) Eds Atherton, M.A., Collins, M.W., and Dayer, M.J. WIT Press $=50$

42. Hunter, P.J., 'Heart models in applied medicine’, Engineering and Technology, (Issue 17, 09 Oct-23 Oct , 2009), pp 16

43. Liu, W.K., Liu,Y., Farrell,D., Zhang,L., Wang, X.S., Fukui, Y., Patankar, N., Zhang, Y., Bajaj, C., Lee, J., Hong, J., Chen,X., and Hsu, H.(2006) Comput Methods Appl Mech Engrg, 195, pp1722 44. Evans, D. J. W., Lawford, P. V., Gunn, J., Walker, D., Hose, D. R., Smallwood, R. H., Chopard, B., Krafczyk, M., Bernsdorf, J., and Hoekstra, A. G. (2008) Philosophical Transactions of the Royal Society A. 336 (1879) pp 3343-.

45. Hoekstra, A., Lorenz, E., Falcone, J., and Chopard, B. (2007) International Journal for Multiscale Computational Engineering 5, pp491. 
46. Liu, W. K. et al. Comput. Methods Appl. Mech. Engrg. 195 (2006) pp1722.

47. Reneman, R.S et al. 'Wall shear stress-an important Determinant of Endothelial Cell Function and Strcture-in the Arterial System in vivo.' (2006) J Vasc Res 43, 251-269

48. Reneman R.S. \& Hoeks A. P.G. 'Wall Shear Stress as measured in vivo: consequences for the design of the arterial system’ (2008) Med Biol Eng Comput 46, 499-507

49. Venneman, P. et al. 'In vivo whole-field blood velocity measurement techniques’ (2007) Exp Fluids 42, 495-511

50. Succi, S. The Lattice Boltzmann Equation for Fluid Mechanics and Beyond, Oxford.

51. Lishchuk, S. V., Halliday, I. and Care, C.M., Phys Rev E 74017701 (2006)

52. Dupin, M. M., Munn, L., Halliday, I., and Care, C. M., Phys. Rev. E 75, 066707 (2007)

53. Spencer, T.J., Pontrelli, G., König, C.S., Collins, M.W. and Halliday, I. In preparation 2010.

54. König, C.S., Long , Q. (2006) 'The influence of the endothelial surface layer on the prediction of wall shear stress in small arteries '. In: J. Biomechanics, Abstracts of the 5th World Congress of Biomechanics, 29 July - 4 Aug., Munich, Germany, 39, Suppl. 1, S445

55. Pontrelli, G., König, C.S., Collins, M.W., Long, Q., Succi, S. Modelling wall shear stress in small arteries using LBM and FVM: the influence of wall shape, see this issue.

56. Reichlin T., Wild A., Dürrenberger M., Daniels A. U., et al. (2005) J. Struct. Biol., 152 pp52-63

57. Walker, D. C., Hill, G., Wood, S. M., Smallwood, R. H., and Southgate, J. (2004) Ieee Transactions on Nanobioscience 3(3), 153-163

58. Walker, D. C., Southgate, J., Hill, G., Holcombe, M., Hose, D. R., Wood, S. M., Mac Neil, S., and Smallwood, R. H. (2004) Biosystems 76(1-3), 89-100

59. Ciofalo, M. et al 'Modelling nanoscale fluid dynamics and transport in physiological flows' (1996) Med Eng Phys, 18,6, 437-451

60. Collins M.W. et al., 'Wall shear stress and arterial performance' submitted for presentation at Bioengineering 09 Conference, Oxford, 24/25 Sept 2009 
61.http://www.biomedtown.org/biomed_town/STEP/Reception/step-

definitions/VirtualPhysiologicalHuman

\section{Figure Captions}

Figure 1 Major components of a healthy artery showing the three wall layers (intima, media and adventitia) and the distribution of endothelial and smooth muscle cells.

Figure 2 a The mechanical environment of the artery showing the distribution of wall stresses $\mathbf{b}$. The influence of shear stress on endothelial cell morphology.

Figure 3 Subject-specific CFD model of the abdominal aorta and the superior mesenteric artery (SMA). a OSI distribution. The region of high OSI (arrowed) at the proximal lip of the outlet of the origin of the SMA from the aorta corresponds with the normal distribution of atheroma reported for this artery. b plots of the different time varying WSS at two locations on the vessel wall. One 
corresponds to an area of high OSI (top), the second to an area of low OSI (bottom).

Figure 4 Evolution of the wall shear stress spatial gradient $[\mathrm{Pa} / \mathrm{m}]$ for an idealised aneurysm with a localized daughter bleb developing on the dome. Development of the aneurysm is accompanied by an increase in the WSSG around its base: this may have significant implications for the degenerative remodeling of the tissue in this region and thus future growth of the aneurysm. At $/ t /=10$, a daughter bleb begins to develop on the aneurysm dome. It can be seen that an annulus of high WSSG forms around the daughter bleb $(/ t /=12)$. This may result in further degeneration of the tissue in this region thus promoting the growth and enlargement (and possibly rupture) of the secondary bleb.

Figure 5 A scale Separation Map (SSM) is a two dimensional map with the horizontal (vertical) axis coding for temporal (spatial) scales. A subsystem occupies a certain area on this map. Arrows indicate coupling between the single scale sub-systems.

Figure 6 The fiction of steady WSS. The magnitude of instantaneous WSS component, $|\nabla u|$, measured in lattice units, plotted over the plane $x-y$ boundary of a rectangular cross-section duct. This data corresponds to a flowing suspension of softened, rigid spheres (see text) at normal haematocrit concentration (40\%), $R e=10$, at the "steady state" of continuum haemodynamics. Flow is induced by a pressure gradient in the $x$-direction. The complex WSS distribution reflects the instantaneous positions of particles immediately above the boundary.

Figure 7. Instantaneous WSS distribution over the endothelial surface for a system comprised of incompressible, Newtonian fluid and a single, advecting "erythrocyte” (modelled here an incompressible, immiscible drop with viscosity contrast and interfacial tension) induced to flow by a pressure gradient in the $\mathrm{x}$-direction. The simulation is symmetric in the upper plane $z=$ constant. The velocity field is periodic in both the $x$ and $y$ directions. The flow data are $R e=1.2$, Weber number $W e=0.225$ and capillary number, $C a=0.1875$ giving an interfacial tension (which controls erythrocyte deformability) of $6.8 \mathrm{mN} / \mathrm{m}$. 
$-23-$ 


\section{Figures}

\section{Figure 1}

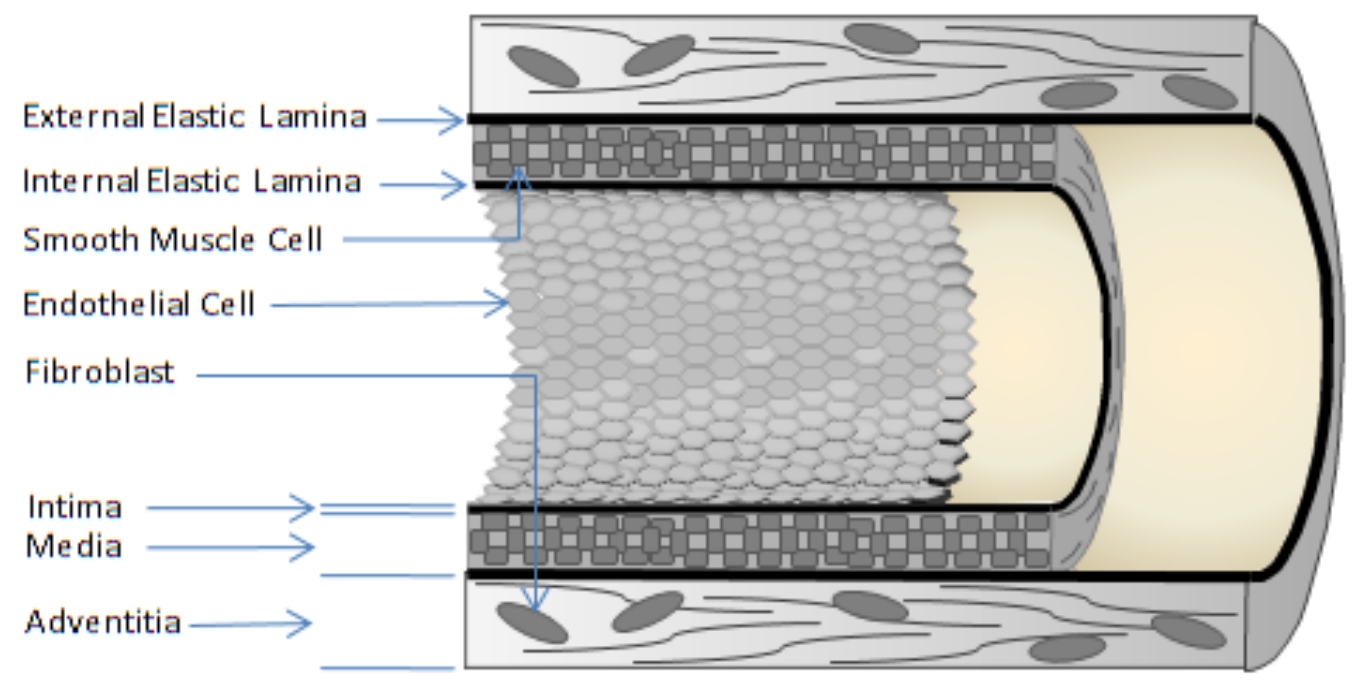


Figure 2 a / b

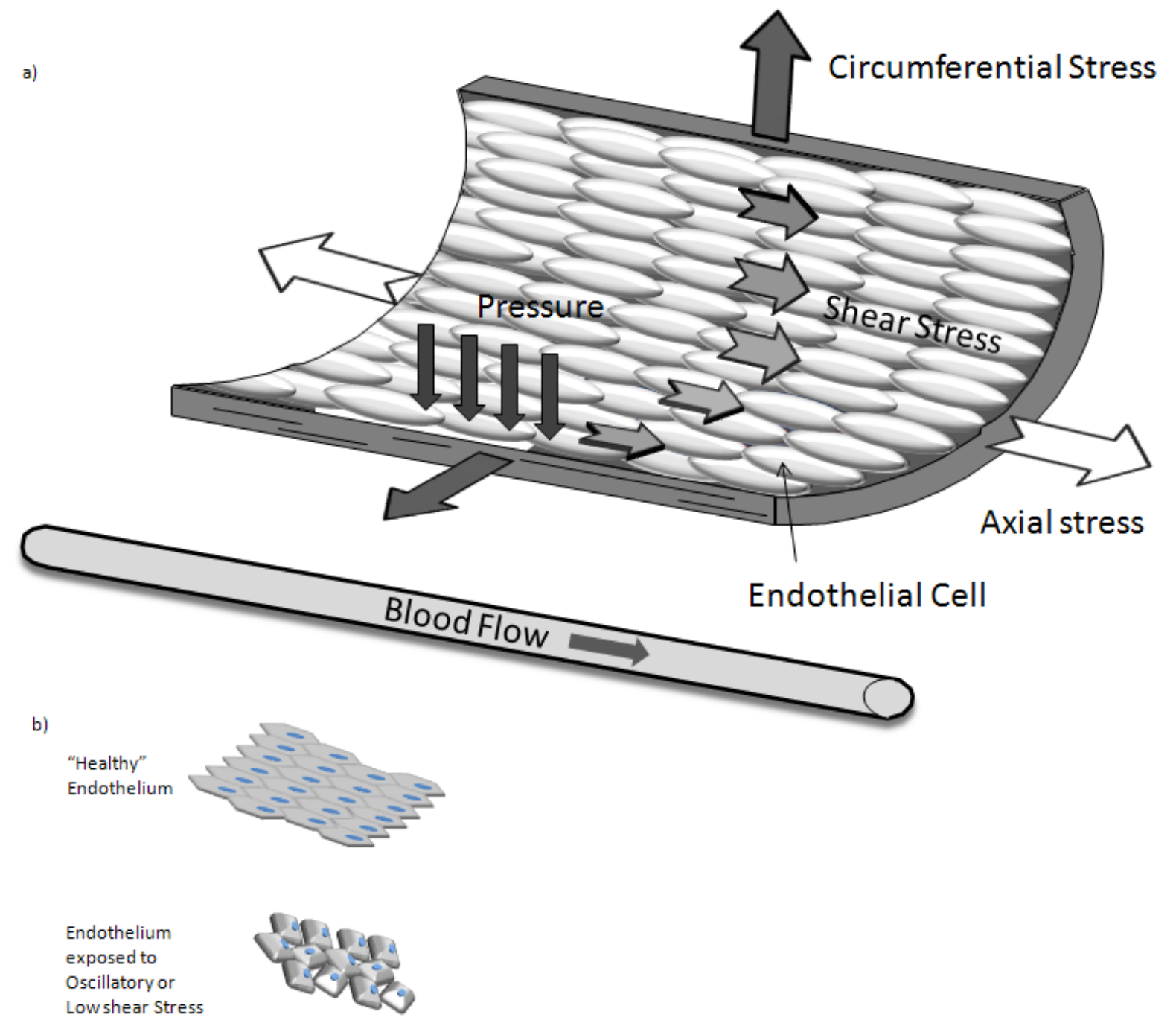


Figure 3

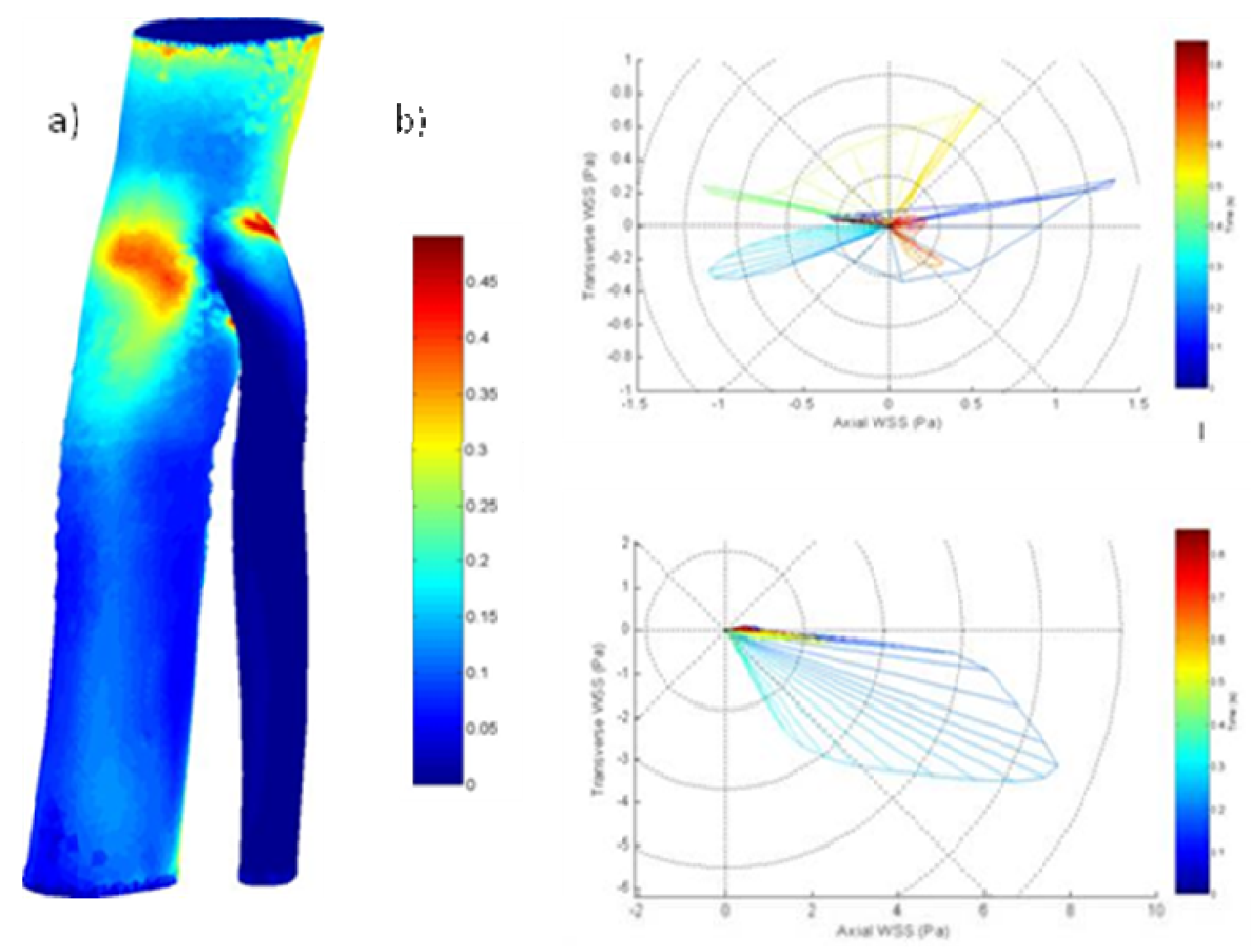

$-26-$ 
Figure 4
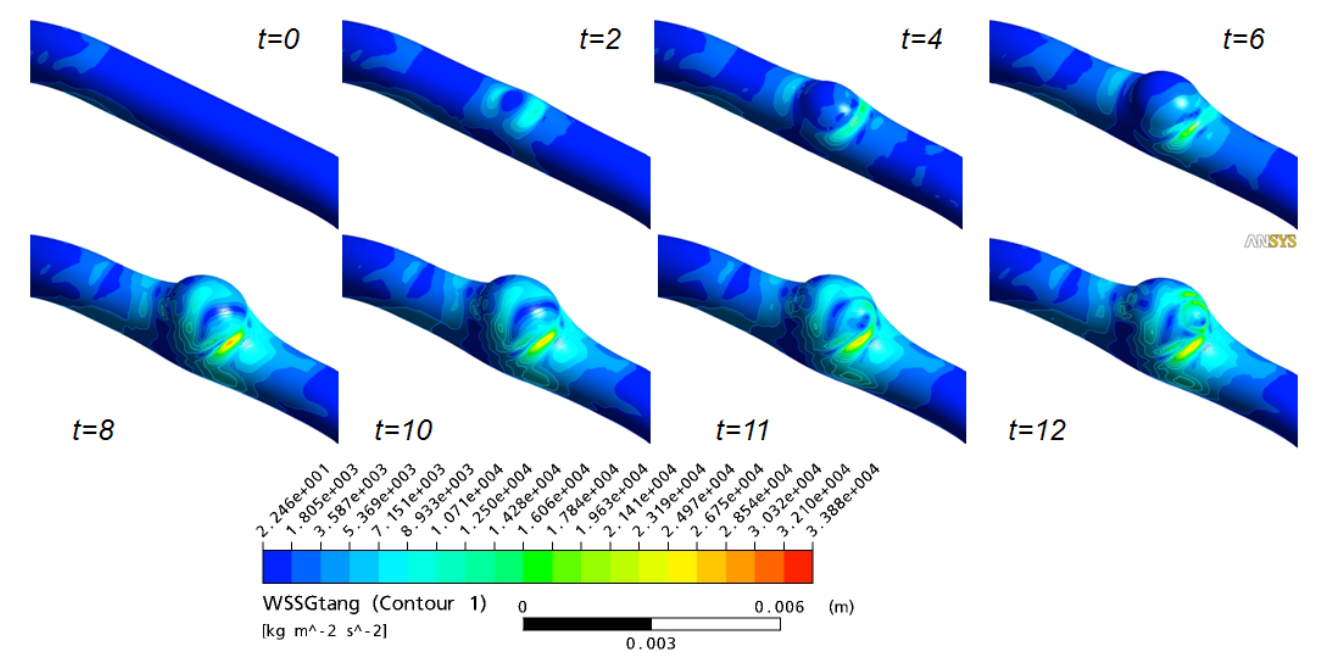

Figure 5

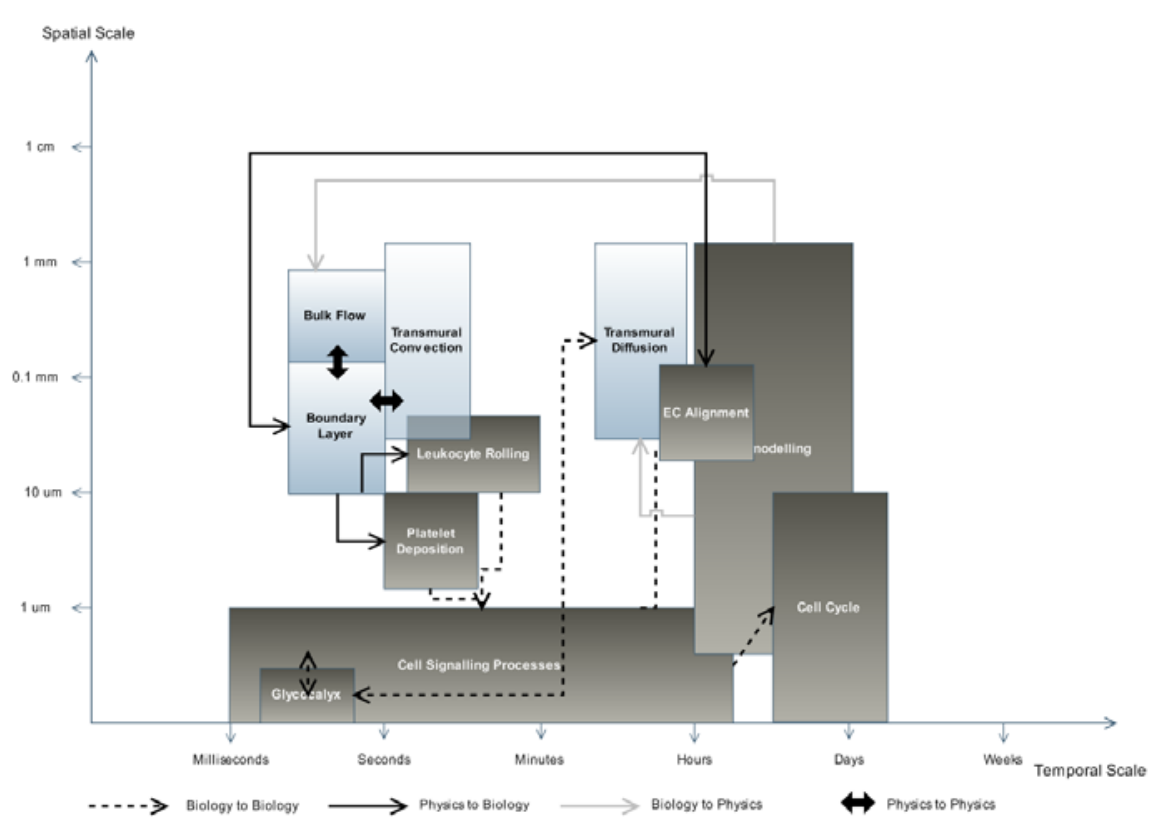


Figure 6

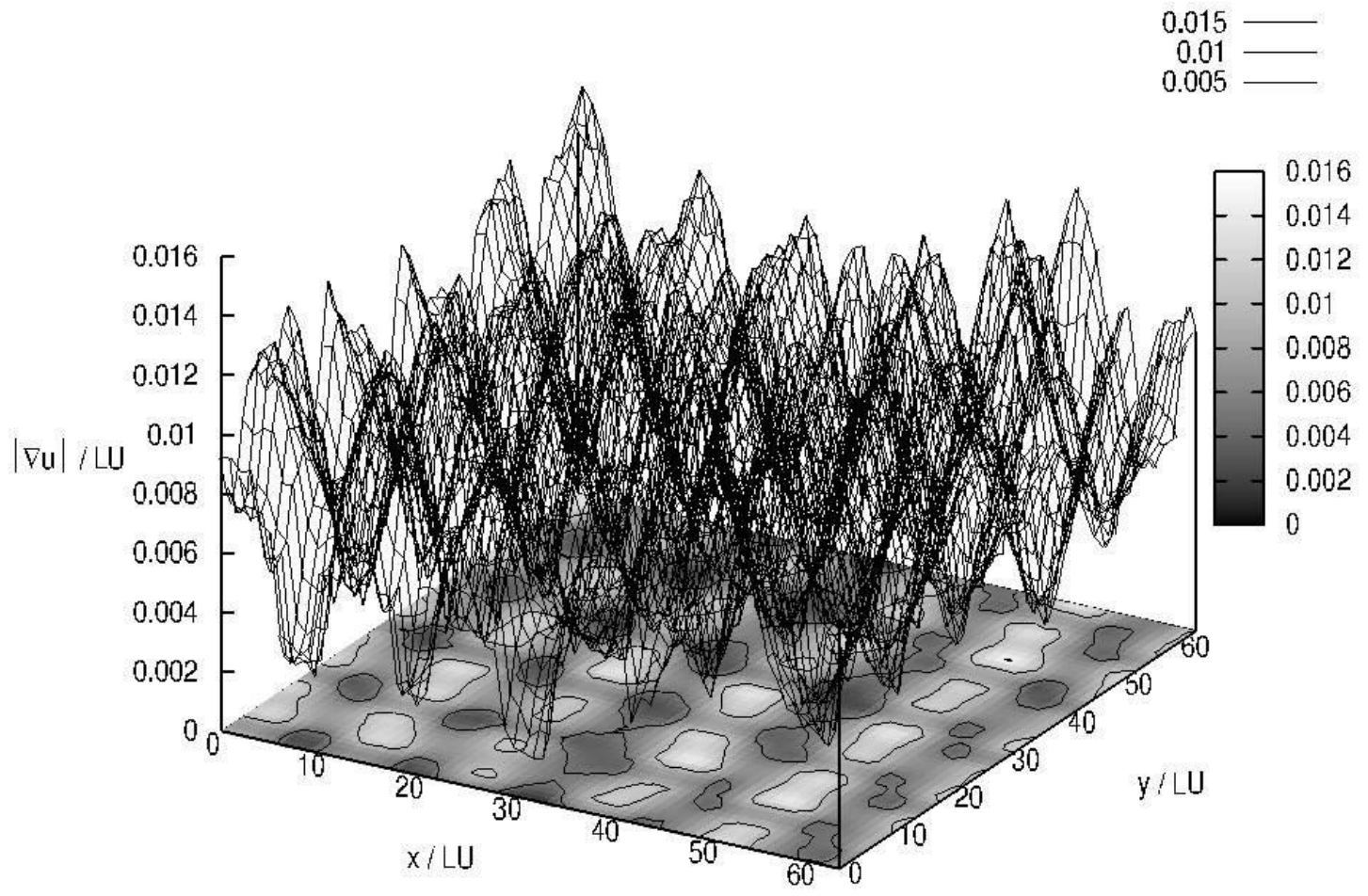


Figure 7

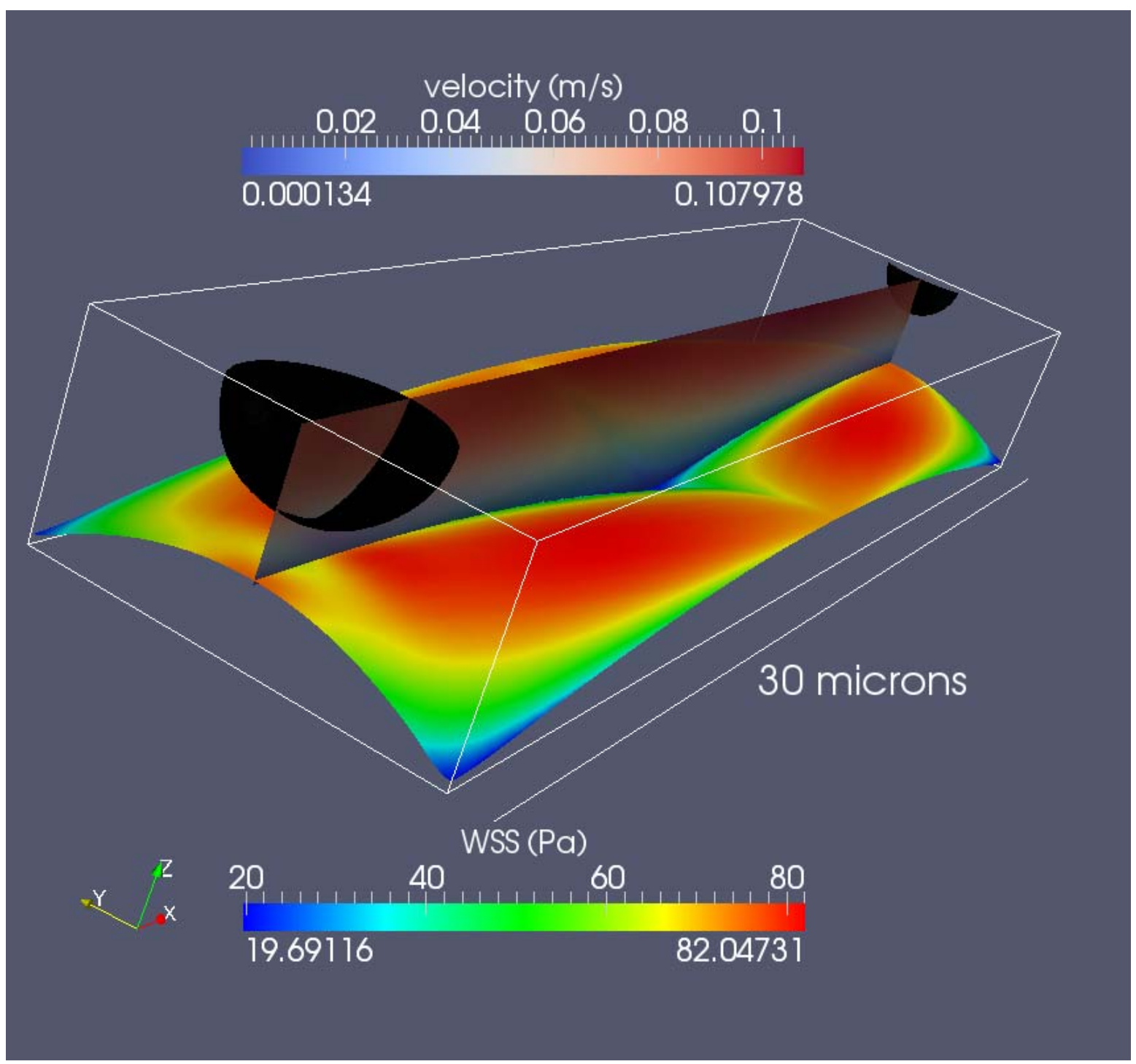

